\title{
Impacts of the ocean lateral diffusion on the El Niño/Southern Oscillation-like variability of a global coupled General Circulation Model
}

\author{
S. Raynaud, S. Speich \\ Laboratoire de Physique des Océans, Brest \\ E. Guilyardi, G. Madec \\ Laboratoire d'Océanographie Dynamique et de Climatologie, Paris
}

\begin{abstract}
A global coupled General Circulation Model is used to investigate effects of the lateral ocean mixing on the amplitude and spectral characteristics of the ENSO-like variability. Three 40-year coupled simulations are analysed. They only differ by the lateral ocean physics parametrized: a horizontal diffusion scheme; an isopycnal scheme ; isopycnal scheme with an Eddy Induced Velocity (EIV) term.

The Multichannel Singular Spectrum Analysis is used to extract linear modes of variability from each simulation and from observations. The strength of the variability, close to that observed in the horizontal and only-isopycnal simulations, is stronger in the two isopycnal configurations, and more especially when the EIV is added. The dominant periods of the variability, also close to those observed, are also affected by the lateral diffusion: the time scale becomes slightly longer as the complexity of the lateral physics increases.
\end{abstract}

\section{Introduction}

El Niño-Southern Oscillation (ENSO) is the largest signal of variability of the tropical climate system. During the last decade, great progress was achieved in modeling ENSO with Coupled ocean/atmosphere General Circulation Models (CGCMs). However, systematic errors persist concerning the mean state and variability [Delecluse et al., 1998]. Coupling Atmospheric GCMs (AGCMs) with Ocean GCMs (OGCMs) are essential in improving these two components: the occurrence of ocean-atmosphere feedbacks can enhance failures that cannot be detected in the forced modes.

Sensitivity to the atmospheric physics has been widely tackled: system behavior intimately depends on the parametrisations and the various schemes used in atmospheric models, such as cloud feedbacks or radiative forcing [Meehl et al., 1982; Stockdale et al., 1994; Ma et al., 1996; Terray, 1998]. Yet, sensitivity studies of CGCM to ocean physics are less numerous [Guilyardi et al., 1999; Hirst et al., 2000]. In this study, we focus our attention on the comparison of three different lateral diffusion schemes for temperature and salinity : the first is a simple horizontal diffusion scheme, the second is an isopycnal scheme and finally the latter scheme with the addition of the EIV term. We focus on how

Copyright 2000 by the American Geophysical Union.

Paper number 1999GL011323.

0094-8276/00/1999GL011323\$05.00 tropical variability of the climate system, and its response in terms of both strength and spectral properties, could be affected by such different ocean physics parametrizations.

To diagnose this variability, the Multichannel Singular Spectrum Analysis (MSSA) [e.g., Plant and Vautard, 1994] is a useful tool.

\section{Model and simulations}

The AGCM is the second version of ARPEGE-Climat [Déqué et al., 1994]. It is a spectral model, used with a T31 truncature giving an equivalent constant horizontal resolution of $3.75^{\circ}$ with 19 vertical levels. The OGCM is OPA [Madec et al., 1998]. Its horizontal resolution is roughly of $2^{\circ} \times 1.5^{\circ}$ [Madec et Imbard, 1996], with an increased meridional resolution to $0.5^{\circ}$ at the equator. The vertical discretization is performed on 31 levels. The two GCMs are coupled via the OASIS coupler [Terray, 1996], without flux correction.

Observations from the ocean seems to indicate that the lateral mixing of tracers, induced by mesoscale turbulence, is preferentially along neutral surfaces. To simulate this effect, the lateral diffusion tensor is rotated from the horizontalvertical axes to the isopycnal-diapycnal axe. No artificial horizontal background mixing is added. In addition, Gent and McWilliams [1990] introduced an extra-advective term on tracer (the EIV), which mimics the effect of potential energy loss associated with baroclinic instability.

Three 40-year coupled simulations [Guilyardi et al., 1999], which only differ in lateral physics, are performed using horizontal diffusion (HOR), isopycnal diffusion (ISO) and an EIV term (ISOG) added to the latter. The same value is used for the eddy coefficient $\left(2000 \mathrm{~m}^{2} / \mathrm{s}\right)$.

\section{The analysis tool}

The fundamental oscillatory nature of ENSO is well described by the "mixed SST-ocean dynamics" mode: this is a stationnary SST-mode oscillating due to wave influences [Neelin et al., 1998]. The dynamics of the modes of variability is essentially linear, becoming more realistic by perturbing it using weak non-linear effects [ $J$ in et al., 1996; Blanke et al., 1997]. As a result, a linear analysis tool (MSSA) can be used to diagnose oscillatory properties of model variability with respect to ENSO.

MSSA can be viewed as an extension of the classical Principal Component Analysis (PCA) where Empirical Orthogonal Functions (EOFs) have a temporal and spatial dimen- 


\section{Sea Level Pressure Sea Surface Temperature}
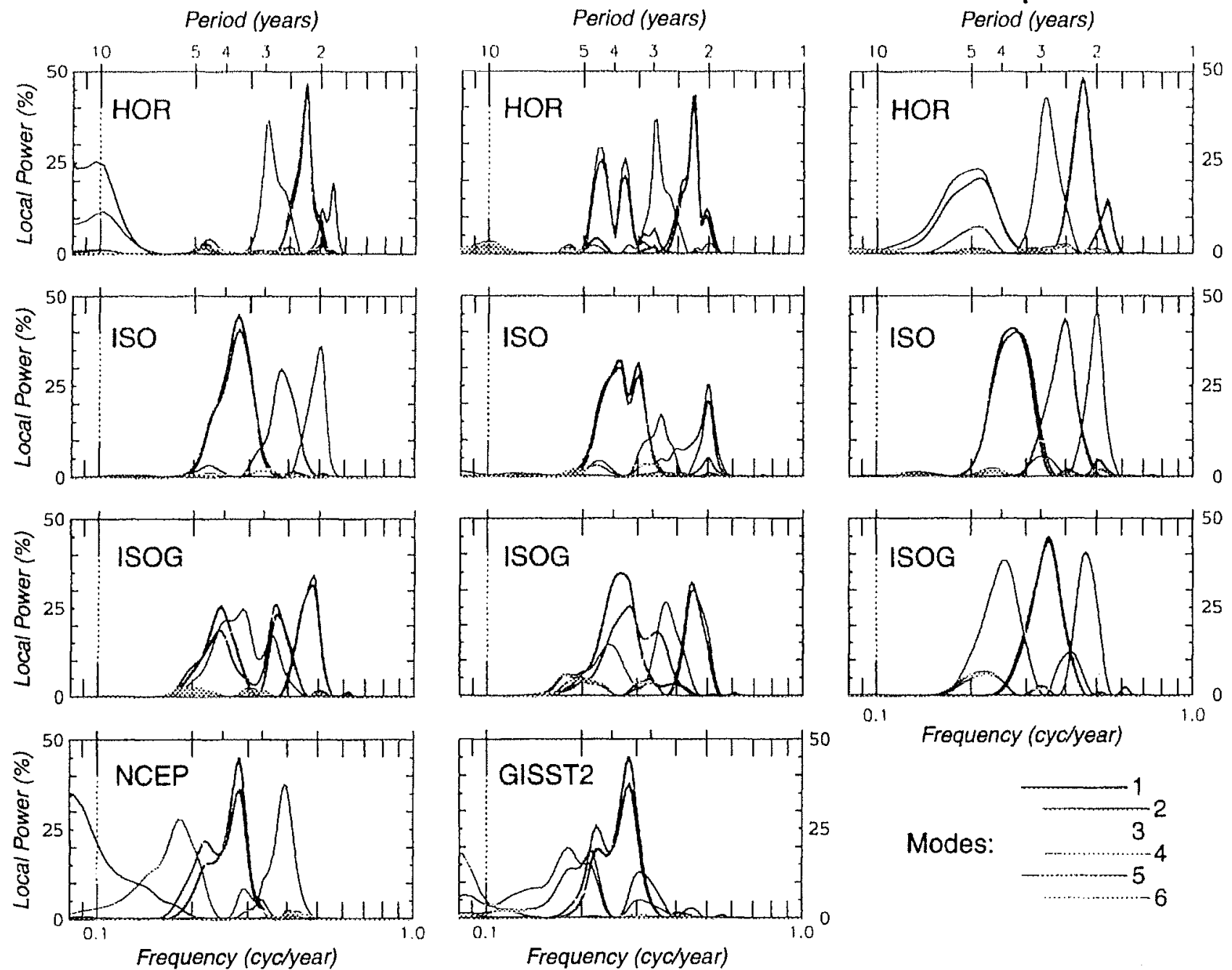

Plate 1. Spectrum of the MSSA modes presented as a relative local power in frequency: a) SLP spectra, for the observations and the three simulations; b) SST spectra ; c) Equatorial Subsurface Temperature Section spectra but without analysis of the observations.

sion. Subsequently, it works as a space-time linear filter, and the resulting modes contain both dimensions. These modes are oscillatory with specific spectral, spatial and propagative properties. The length of EOFs (window) is the analysis parameter. This method is particularly efficient in extracting oscillations from short noisy signals (except colored noise as it uses covariances). Each oscillation is composed of a sum of two identical modes (pair) with their respective principal component and EOF in phase quadrature to each other. In addition, since we are looking for oscillations in the coupled system, similar modes of variability (spectrally speaking) should be found in most variables of both the atmospheric and ocean model, in the space domain of interest. This is the main criterion used to select pairs relevant to the coupled behavior.

Samples are constructed with monthly averaged values. In order to omit the high frequency variability, an 11-month gaussian filter is preliminary applied. After removing the mean, the annual cycle and linear seasonal trend are eliminated. MSSA is computationally very expensive. Consequently, the number of statistical degrees of freedom is re- duced with a simple PCA. Typically, the first 20 EOFs are kept (this represents approximately $90 \%$ of the total variance). Results presented here have been found to be insensitive to the number of EOFs kept. 'The analysis domain extends spatially from $30^{\circ} \mathrm{S}$ to $30^{\circ} \mathrm{N}$ over the Pacific Ocean.

\section{Results}

Some biases of the atmospheric model affect the spatial structure of the coupled mean state [Guilyardi, 1997], and therefore also have an impact on the ENSO-like variability: simulated El Niño is too symmetric with respect to the equator and extends too far in the West; the coastal El Niño is missing. However, the ENSO-like signal is strong enough to be diagnosed in terms of amplitude and spectral characteristics thanks to the MSSA that naturally adapts its filtering to the spatial structure of the variability.

The three simulations (HOR, ISO and ISOG) undergo a drift during approximately the first 15 years before a different steady state for each simulation is reached. If we interpret the variability of the system as oscillations around a 
Table 1. Periods (in years) of the first robust pairs extracted by the MSSA, for the observations and the three coupled simulations.

\begin{tabular}{ccccc} 
Pairs & Obs. & HOR & ISO & ISOG \\
\hline 1 & 3.5 & 2.2 & 3.6 & 2.8 \\
2 & 4.6 & 2.9 & 2.0 & 3.9 \\
3 & 2.5 & 1.8 & 2.7 & 2.2 \\
4 & $\cdots$ & 4.6 & 1.3 & 1.8 \\
\hline
\end{tabular}

mean state, we expect to find a different resulting variability for each run. The short temporal extent of the simulations prevent the equatorial region being influenced by the low frequency variability of higher latitudes.

The Sea Surface Temperature (SST) averaged in the box $\left[150^{\circ}-90^{\circ} \mathrm{W}, 5^{\circ} \mathrm{S}-5^{\circ} \mathrm{N}\right]$, also called NIÑO3 index, is a good indicator of the ENSO-like variability amplitude. We compare the model behavior with the GISST2 data sets [Rayner et al., 1996], for the 1958-1997 period (40 years as for the simulations length). Other comparisons are performed using the Southern Oscillation Index (SOI), which is approximately the cross Pacific Sea Level Pressure (SLP) difference (East minus West). For this, we used the SLP data set provided by the NCEP reanalysis [Kalnay et al., 1996].

Indices computed from unfiltered values may be contaminated by unexpected modes of variability such as noise, trends or very important, and thus instantaneously more non-linear, ENSO events. If ENSO was a completely linear phenomenon in the presence of a regular forcing, it should be approximated by a regular (growing or decaying) oscillation. Presence of weak non-linearities perturb, in various ways, this single oscillatory mode. Coexistence of multiple quasi-linear oscillatory modes must be expected. The MSSA of SLP and SST filters out a few oscillations (pairs) : plate 1 displays the spectra computed with the Maximum Entropy Method [Plaut and Vautard, 1994] of the first 6 modes for the observations and the three simulations. A spectral window of $10 \mathrm{y}$ was used, and the results are quite insensitive to this choice. The most powerful pairs and corresponding periods are listed in table 1.

Since statistical confidence on spectral characteristics is low for $40 y$ data sets, no precise conclusion is given for the dominant periods. All the periods fall in the common range of ENSO ( $\sim 2$ to 6 years). In the observations, the $3.5 y$ and $2.5 y$ oscillations may be related to the Quasi-Quadriennal and Quasi-Biennal oscillations, respectively [Jiang et al., 1995]. As for the observations, the Equatorial Subsurface Temperature Section (ESTS) spectra are also given for the simulations and appear less noisy. Certainlym this is due El Niño being an almost equatorial phenomenon in the model. By simply weighting the principal periods by the variance

Table 2. Variances of NIÑO3 $\left({ }^{\circ} \mathrm{C}^{2}\right)$, SOI $\left(h \mathrm{~Pa}^{2}\right)$ and ESTSI $\left({ }^{\circ} \mathrm{C}^{2}\right)$

\begin{tabular}{lccc}
\hline & NIÑO3 & SOI & ESTSI \\
\hline Obs. & 0.27 & 0.52 & $\ldots$ \\
HOR & 0.12 & 0.41 & 0.43 \\
ISO & 0.23 & 0.95 & 0.96 \\
ISOG & 0.37 & 1.27 & 1.82 \\
\hline
\end{tabular}

of their respective mode, the averaged period is found to be similar for ISO and ISOG (respectively $3.1 \mathrm{y}$ and $3.2 \mathrm{y}$ ) and smaller for HOR (2.7y).

Since only the ocean model is tuned, another index is computed by averaging the ESTS values within a box of the eastern thermocline (where the anomaly is maximum). This is refered as to ESTSI. All the powerful oscillations found in the common ENSO frequency range ( 3 to 5 pairs) are added to compute the three indices. Table 2 summarizes the results of these indicators for the three simulations together with those derived from observations. Results for a window set to $10 \mathrm{y}$ and $15 \mathrm{y}$ have been averaged. A comparison to observations indicates that only the variance of ISOG seems to be clearly different (greater), the variance of HOR and ISO remaining closer to the real one. It is obvious that the variability diagnosed in $\mathrm{NINO} 3$ is greater in the ISO and ISOG than in HOR, and is enhanced with the addition of an EIV term. Use of the only-isopycnal scheme almost doubles the variance (from 0.12 to 0.23 ). A similar increase is obtained with the EIV term (from 0.23 to 0.37). The analysis of SOI corroborates these results: as for NIÑO3, approximately the same factors allow to pass from one simulation to the other ( $\sim 2$ from HOR to ISO and $\sim 1.3$ from ISO to ISOG). The subsurface index (ESTSI) reinforces the appearant increase in variance with increased lateral physics complexity. A Kolmogorov-Smirnov test used to compare the distribution of extrema of ocean indices of the three simulations, shows that the confidence level lies between $94 \%$ and $99 \%$ for NIÑO3 and it is always greater than $99 \%$ for ESTSI. Thus good confidence can be given to the differences in variances between each simulation.

Despite slight increase in period and amplitudes, the model results are close to observed ones. We tsuggest that this coupled model was able to capture the main physical patterns of ENSO (even if slight differences may affect its prediction).

It appears that the variability is strengthened by the addition of lateral ocean physics (isopycnal diffusion and EIV term) and that a slight shift to lower typical frequencies occurs.

\section{Conclusion}

The sensitivity of ENSO-like variability of a CGCM to its ocean lateral diffusion has been investigated. This is achieved by performing three simulations in which horizontal, isopycnal, and isopycnal with EIV diffusion are used. A strengthening of the variability and a slight red shift occurs with increased lateral physics complexity.

These results emphasize the role of sub-meso-scale physics on low frequency variability of the tropical climate.

Possible coherent explanations for the increase in variance are found by involving the subtle impact of three parametrisations on the mean equatorial upwelling and stratification. This clearly controls the growth of anomalies. However, more work is needed to improve and confirm such explanations.

Acknowledgments. We gratefully thanks B. Blarke, E. da Costa, P. Delecluse, G. Plaut, M. Ghil and especially the anonymous reviewer for their help and fructifull comments. GISST2 data set provided through the UKMO Hadley Center. NCAR/NCEP Reanalysis data provided through the NOAA Climate Diagnostics Center. 


\section{References}

Blanke, B.,J. D. Neelin, and D. Gutzler, Estimating the effect of the stochastic wind stress forcing on ENSO irregularity, J. Clim., 10, 1473-1486, 1997.

Déqué, M., C. Dreveton, C. Braun, and D. Cariolle, The climate version of Arpege/IFS: a contribution to the French community climate modeling, Clim. Dyn., 10, 249-266, 1994.

Delecluse, P., M. K. Davey, Y. Kitamura, S. G. H. Philander, M. Suarez, and L. Bengtsson, Coupled general circulation modeling of the tropical Pacific, J. Geophys. Res., 103, 14357-14373, 1998.

Gent, P. R., and J. C. McWilliams, Isopycnal mixing in ocean circulation models, J. Phys. Oceanogr., 20, 150-155, 1990.

Guilyardi, E., Rôle de la physique océanique sur la formation/consommation des masses d'eau dans un modèle couplé ocan-atmosphère, Ph.D thesis, Université Paul Sabatier Toulouse, pp. 195, 1997.

Guilyardi, E., G. Madec, and L. Terray, The role of lateral ocean physics in the upper thermal balance of a coupled oceanatmosphere GCM, Note du pôle de modélisation, 13, IPSL, France, pp. 11, 1999.

Hirst, A. C., S. P. O'Farrel, and H. B. Gordon, Comparison of a Coupled Ocean-Atmosphere Model with and without oceanic Eddy-Induced Advection. Part I: Ocean spinup and control integrations, Journal of Climate, 13, 139-163, 2000.

Jiang, N., J. D. Neelin, and M. Ghil, Quasi-quadriennal and quasi-biennal variability in the equatorial Pacific, Clim. Dyn., 12, 101-112, 1995.

Jin, F.-F., J. D. Neelin, and M. Ghil, EI Niño/Southern Oscillation and the annual cycle: Subharmonic frequency locking and aperiodicity, Physica D, 98,442-465, 1996.

Kalnay, E., et al., The NCEP/NCAR 40-Year Reanalysis Project, Bull. Amer. Meteor. Soc., 77, 437-471, 1996.

Lazar, A., G. Madec and P. Delecluse, A rationalization of the Veronis upwelling / downwelling system and its sensitivity to mixing parametrization in an idealized OGCM, J. Phys. Oceanogr, in press, 1999.

Ma, C.-C., R. Mechoso, A. W. Robertson, and A. arakawa, Peruvian stratus clouds and the tropical Pacific circulation GCM study, J. Clim., 9, 1635-1645, 1996.

Madec, G., P. Delecluse, M. Imbard, and C. Levy, OPA version 8.1 Ocean General Circulation Model Reference Manual, Note du pôle de modélisation, 11, IPSL, Prance, pp. 91, 1998.
Madec, G., and M. Imbard, A global ocean mesh to overcome the North Pole singularity, Clim. Dyn., 12, 381-388, 1996.

Meehl, G. A., W. M. Washington, and A. J. Semtner, Experiments with a global ocean model driven by observed atmospheric forcing, J. Phys. Oceanogr., , 12, 301-312, 1982.

Neelin, J. D., D. S. Battisti, A. C. Hirst, F..F. Jin, Y. Wakata, T. Yamagata and S. E. Zebiak, ENSO theory, J. Geophys. Res., 103, 14261-14290, 1998.

Plaut, G., and R. Vautard, Spells of Low-Frequency Oscillations and Weather Regimes in the Northern Hemisphere, J. Atmos. Sci., 2, 210-236, 1994 .

Rayner, N., E. Horton, C. Parker, R. and Hackett, Version 2.2 of the global ice ans sea surface temperature data set, Tech. Rep. 74, 35 pp., Hadley Centre Climate Research, 1996.

Robertson, A. W., C.-C. Ma, C. R. Mechoso, and M. Ghil, Simulation of the tropical-Pacific climate with a coupled oceanatmosphere GCM, Part II: Interannual variability, J. Clim., 8, 1199-1216, 1994

Stockdale, T. N., D. Anderson, M. K. Davey, P. Delecluse, A Kattenberg, Y. Kitamura, M. Latif, and T. Yamagata, Intercomparison of tropical Pacific Ocean GCM's, Tech. Rep. WMO/TD-545, 90 pp., World Meteorol. Orgn., Geneva, 1993.

Stockdale, T. N., M. Latif, G. Burgers, and J.-O. Wolff, Some sensitivities of a coupled ocean-atmosphere GCM, Tellus, Ser. A, 46, 367-380, 1994.

Terray, L., The OASIS Coupled User Guide Version 2.1, Tech. Rep. TR/CMGC/96-46, CERFACS, 1996.

Terray, L., Sensitivity of climate drift to atmospheric parametrization in a coupled ocean-atmosphere general circulation model, J. Clim., 11, 1633-1658, 1998.

S. Raynaud and S. Speich, Laboratoire de Physique des Océans (UBO/CNRS/IFREMER), Université de Bretagne Occidentale, 6 av. Le Gorgeu - BP 809, 29285 Brest Cedex, France. (e-mail: raynaud univ-brest.fr)

E. Guilyardi and G. Madec, Laboratoire d'Océanographie Dynamique et de Climatologie (UPMC/CNRS/IRD), Université Pierre et Marie Curie, Boite 100, 4 place Jussieu, 75252 Paris Cedex, France.

(Received December 30, 1999; revised March 23, 2000; accepted April 28, 2000.) 